\title{
No difference in high-magnification morphology and hyaluronic acid binding in the selection of euploid spermatozoa with intact DNA
}

\begin{abstract}
Suchada Mongkolchaipak ${ }^{1}$ and Teraporn Vutyavanich ${ }^{2}$
In this study, we compared conventional sperm selection with high-magnification morphology based on the motile sperm organellar morphology examination (MSOME) criteria, and hyaluronic acid (HA) binding for sperm chromosome aneuploidy and DNA fragmentation rates. Semen from $\mathbf{5 0}$ severe male factor cases was processed through density gradient centrifugation, and subjected to sperm selection by using the conventional method (control), high magnification at $\times 6650$ or HA binding. Aneuploidy was detected by fluorescence in situ hybridization with probes for chromosomes $13,18,21, \mathrm{X}$ and $\mathrm{Y}$, and DNA fragmentation by the terminal deoxynucleotidyl transferase dUTP nick end labelling (TUNEL) method. Spermatozoa selected under high-magnification had a lower DNA fragmentation rate ( $2.6 \%$ vs. $1.7 \% ; P=0.032)$, with no significant difference in aneuploidy rate $(0.8 \%$ vs $0.7 \% ; P=0.583)$, than those selected by the HA binding method. Spermatozoa selected by both methods had much lower aneuploidy and DNA fragmentation rate than the controls ( $7 \%$ aneuploidy and $26.8 \%$ DNA fragmentation rates, respectively). In the high-magnification group, the aneuploidy rate was lower when the best spermatozoa were selected than when only the second-best spermatozoa were available for selection, but the DNA fragmentation rate was not different. In conclusion, sperm selection under high magnification was more effective than under HA binding in selecting spermatozoa with low DNA fragmentation rate, but the small difference $(0.9 \%)$ might not be clinically meaningful. Both methods were better than the conventional method of sperm selection.
\end{abstract}

Asian Journal of Andrology (2013) 15, 421-424; doi:10.1038/aja.2012.163; published online 25 February 2013

Keywords: aneuploidy; DNA fragmentation; hyaluronic acid binding; intracytoplasmic sperm injection (ICSI); intracytoplasmic morphologically selected sperm injection (IMSI); motile sperm organellar morphology examination; physiologic intracytoplasmic sperm injection

\section{INTRODUCTION}

Intracytoplasmic sperm injection (ICSI) is a very effective method for the treatment of severe male factor infertility. It allows the use of a single motile spermatozoon to fertilise an oocyte. ${ }^{1}$ The technique is so effective that fertilization of an oocyte can be achieved even with the use of a spermatozoon with severe DNA fragmentation. ${ }^{2}$

Men with severe male factor infertility are known to have a higher frequency of sperm aneuploidies and DNA fragmentation than fertile men. ${ }^{3-8}$ Although the consequences of inseminating oocytes with abnormal spermatozoa are not known for certain, there is increasing evidence that such circumstances may cause poor fertilization, defective pre-implantation embryonic development, and high rates of miscarriage and morbidity in the offspring, including childhood cancer. ${ }^{8}$ One major challenge ICSI facing is, therefore, the selection of the best spermatozoon for micro-insemination.

Many studies have shown that intracytoplasmic morphologicallyselected sperm injection (IMSI) can significantly increase the fertilization and pregnancy rate of ICSI, while decreasing its abortion rate. ${ }^{9-11}$ The disadvantage of IMSI is that it requires expensive equipment, as well as expertise and time, to select the spermatozoa under high-power magnification. A much less expensive and less labour-intensive alternative to select the best spermatozoa for ICSI is hyaluronic acid (HA) binding. ${ }^{12-15}$ One such commercial product includes a Petri dish coated with HA, called the "Petridish ICSI" to perform the physiologic intracytoplasmic sperm injection (PICSI). ${ }^{15}$ Parmegiani et al. ${ }^{15}$ showed that nuclear normality, according to the motile sperm organellar morphology examination (MSOME) criteria, was significantly higher in HA-bound than in unbound spermatozoa.

The primary objective of this study was to compare the effectiveness of both methods in selecting spermatozoa with lower aneuploidy and DNA fragmentation rates. Obviously, if both methods give comparable results, HA binding will be the method of choice as it is easier, less time-consuming and less expensive.

\section{MATERIALS AND METHODS}

Collection and preparation of semen samples

The Ethics Committee of Phyathai Sriracha Hospital approved this study. Semen samples were provided by male partners of couples, who 
gave written informed consent for the use of their spermatozoa for research. These couples presented themselves for ICSI treatment because of severe male factor infertility (sperm concentration $<5$ million $\mathrm{ml}^{-1}$ on at least two occasions, 4 weeks or more apart). Patients were excluded if they had abnormal karyotype or had a previous history of treatment with cytotoxic drugs or radiotherapy to the genital area, or were currently using antioxidants or hormone therapy in 3 months before the study.

The samples were collected by masturbation after 48- to 72-h abstinence. After liquefaction, standard semen analysis was performed within 1-h collection, according to the guideline described by the World Health Organization. ${ }^{16}$ Semen samples were layered on top of $90 \%$ / $40 \%$ Sil-select (FertiPro, Beernem, Belgium) gradients, and centrifuged at $375 \mathrm{~g}$ for $20 \mathrm{~min}$ at $25^{\circ} \mathrm{C}$. The sperm pellet was then washed twice in sperm washing medium, supplemented with $0.5 \%(\mathrm{w} / \mathrm{v})$ human serum albumin (HSA), at $300 \mathrm{~g}$ for $5 \mathrm{~min}$. The final pellet was suspended in $0.2 \mathrm{ml}$ sperm washing medium, supplemented with $0.5 \%$ HSA. The remaining washed spermatozoa after ICSI were used in the study.

\section{Method of sperm selection}

In the control group, a drop of sperm suspension was added to a drop of $8 \%(\mathrm{w} / \mathrm{v})$ polyvinylpyrrolidone solution under sterile paraffin oil in an ICSI dish. An embryologist selected spermatozoa with normal appearance in the conventional way with an inverted microscope, equipped with Hoffman modulation contrast and a heated stage, at a magnification of $\times 400$. An ICSI pipette was used to transfer the selected spermatozoa and pool them in a small drop of fertilization medium under oil.

In the high-magnification group, the sperm suspension was transferred to a PVP drop on a sterile glass dish (Fluorodish, World Precision Instrument, Sarasota, FL, USA), covered with paraffin oil. The spermatozoa were assessed at room temperature, based on MSOME criteria, using an inverted microscope (Leica DMI6000B, Wetzlar, Germany) equipped with high power differential interference contrast optics to achieve a final electronic magnification of $\times 6650$, as described by Peer et al. ${ }^{17}$ At least 20 spermatozoa, classified as normal according to MSOME criteria (smooth, symmetric and oval configuration of the sperm head with an average length of $4.75 \pm 0.28 \mu \mathrm{m}$ and average width of $3.28 \pm 0.20 \mu \mathrm{m}$, normal acrosome and postacrosomal lamina with normal neck and tail, and no extrusion or invagination of the nuclear chromatin mass), with small nuclear vacuoles occupying less than $4 \%$ of the nuclear area, ${ }^{9,18}$ were collected in a small drop of fertilization medium. In the cases where the best spermatozoa could not be found, the second-best spermatozoa (those with a single specific nuclear malformation and without a large nuclear vacuole) were chosen as described by Berkovitz et al. ${ }^{19}$

In the PICSI group, a Petri dish coated with HA (Biocoat, Fort Washington, PA, USA) was used for sperm selection, as described by Huszar et al. ${ }^{12}$ In brief, a drop of sperm suspension was placed close to the edge of the HA spot, and the spermatozoa were allowed to migrate to the drop of HA. After 15 min incubation, only the spermatozoa that were bound to the HA spot and exhibited vigorous tail beating were selected. At least 100 bound spermatozoa were removed one-by-one using an ICSI pipette, and transferred into a small drop of fertilization medium.

\section{Detection of sperm DNA fragmentation}

DNA fragmentation was analysed by the terminal deoxyribonucleotidyl transferase-mediated dUTP nick end labelling (TUNEL) assay, by using an in situ cell-death detection kit (Roche Diagnostic, Mannheim, Germany). The method was carried out according to the manufacturer's instructions.
Selected spermatozoa were transferred into small drops of phosphate-buffered saline (PBS) on pre-cleaned colour-frost plus microscopic slides. The slides were air-dried at room temperature for at least $5 \mathrm{~h}$ and fixed in cold $\left(4{ }^{\circ} \mathrm{C}\right)$ Carnoy's solution (methanol: glacial acetic acid, $3: 1$ ) for $15 \mathrm{~min}$. The slides were then permeabilized with $0.1 \%(\mathrm{v} / \mathrm{v})$ Triton $\mathrm{X}-100$ in $0.1 \%(\mathrm{w} / \mathrm{v})$ sodium citrate at $4{ }^{\circ} \mathrm{C}$ for $2 \mathrm{~min}$. After being washed with PBS, the slides were incubated with TUNEL reaction mixture containing $0.5 \mathrm{IU} \mu \mathrm{l}^{-1}$ terminal deoxynucleotidyl transferase and fluorescein-dUTP for $1 \mathrm{~h}$ in a humid chamber at $37^{\circ} \mathrm{C}$. After the enzyme reaction was stopped, the slides were washed with PBS, air-dried, and then counterstained with Vectashield Mounting medium (Vector Laboratories, Burlingame, CA, USA), containing $1.5 \mu \mathrm{g} \mathrm{ml}^{-1} 4,6$ diamidino-2-phenylindole (DAPI).

The final evaluation was performed with a fluorescence microscope (Zeiss AxioImager Z1, Carl Zeiss, Berlin, Germany), equipped with an automated cell counter system (Metasystem, Altlussheim, Germany). The positive control utilized fixed sperm slides from a male with normal sperm count, previously incubated with DNase $\mathrm{I}\left(1 \mathrm{U} \mathrm{ml}{ }^{-1}\right)$ (New England Biolabs Inc., Ipswich, MA, USA) for $15 \mathrm{~min}$ at $37^{\circ} \mathrm{C}$. They exhibited an $89 \%-98 \%$ rate of DNA fragmentation. Spermatozoa with DNA fragmentation (positive cells) exhibited green fluorescence while TUNEL-negative cells showed blue fluorescence of 4,6 diamidino-2-phenylindole in the background. Negative control slides were those from which the enzyme terminal transferase had been omitted, and showed no green fluorescence.

\section{Detection of sperm chromosome aneuploidy, by the five-colour} fluorescence in situ hybridization technique

After completion of the TUNEL assay, the coverslips were gently removed. The slides were washed with PBS at $37{ }^{\circ} \mathrm{C}$, air dried, and dehydrated in $70 \%, 90 \%$ and $100 \%$ ethanol for 5 min each. They were then air-dried for $20 \mathrm{~min}$ at room temperature, and stored at $-20{ }^{\circ} \mathrm{C}$ until analysis.

Sperm nuclear decondensation was performed according to the method described by Vidal et al. ${ }^{20}$ In brief, the slides were exposed to $5 \mathrm{mmol} \mathrm{l}^{-1}$ dithiothreitol plus $0.1 \mathrm{~mol} \mathrm{l}^{-1}$ Tris/ $1 \%$ (v/v) Triton-X for at least $12 \mathrm{~min}$ at $37{ }^{\circ} \mathrm{C}$, and then immediately transferred to $2 \times$ strength saline-sodium citrate buffer for $3 \mathrm{~min}$ at room temperature, and dehydrated in an ethanol series of $70 \%, 90 \%$ and $100 \%$ for 2 min each. The slides were then air-dried at room temperature.

After nuclear decondensation, the slides were hybridized with MultiVysion pre-implantation genetic probes (Vysis, Downers Grove, IL, USA), according to the manufacturer's instructions. The kit consisted of five-colour probes for chromosomes 13, 18, 21, X and Y. The probe cocktail $(3 \mu \mathrm{l})$ was added to the target area on each slide and covered with a 12-mm circular glass coverslip. The coverslip was covered with Parafilm to prevent humidity loss. Slides were heated to $73{ }^{\circ} \mathrm{C}$ for $5 \mathrm{~min}$ and then incubated at $37{ }^{\circ} \mathrm{C}$ for $4 \mathrm{~h}$ in a Hybrite incubation chamber. Hybridized slides were washed in $0.4 \times$ strength saline-sodium citrate buffer $/ 0.3 \% \mathrm{NP}-40$ at $73{ }^{\circ} \mathrm{C}$ for 2 min and then in $2 \times$ strength saline-sodium citrate buffer $/ 0.1 \% \mathrm{NP}-40$ at room temperature for $1 \mathrm{~min}$. Slides were air-dried in the dark, and then mounted along with Antifade II solution (Vysis). The coverslips were sealed with clear nail polish for semipermanent mounting. Lymphocytes from a male with normal karyotype were used as controls and showed $>98 \%$ hybridization frequency.

Slides were examined with a Zeiss fluorescence microscope (Axioimager Z1) equipped with automatic cell counter and appropriate filter sets. Fluorescence in situ hybridization analysis was automated 
Table 1 Summary of analysis and the number of spermatozoa with aneuploidy and DNA fragmentation

\begin{tabular}{lcccc}
\hline & Control & HA-binding & $\times 6650+$ MSOME & P \\
\hline Total spermatozoa analysed & 10067 & 9825 & 1745 & - \\
Spermatozoa with DNA fragmentation & 2706 & $260^{\mathrm{a}}$ & $31^{\mathrm{a}}$ & 1.7 \\
DNA fragmentation rate (\%) & 26.8 & 2.6 & $12^{\mathrm{b}}$ & $<0.001$ \\
Spermatozoa with aneuploidy & 706 & $80^{\mathrm{b}}$ & 0.7 & - \\
Aneuploidy rate (\%) & 7 & 0.8 & - & $<0.001$ \\
\hline
\end{tabular}

Abbreviation: $\mathrm{HA}$, hyaluronic acid; MSOME, motile sperm organellar morphology examination.

${ }^{\text {a }} P=0.032,{ }^{\mathrm{b}} P=0.583$, compared with high magnification $\times 6650+$ MSOME criteria (chi-squared).

by the standard Metafer system and the Metacyte imaging software (Metasystem, Altlussheim, Germany). Manual analysis of the fluorescence signals was performed at $\times 100$ under oil to decrease the false positive and negative rates Spermatozoa were scored according to previously-described criteria. ${ }^{21} \mathrm{~A}$ disomic spermatozoon displayed two clear fluorescence signals for the same chromosome, which were similar in size and intensity, and were more than one domain (signal diameter) apart. Diploid spermatozoon showed two fluorescence signals for both autosomes and sex chromosomes. The absence of the fluorescence signal for any chromosome implied nullisomy for that chromosome.

\section{Data collection and statistical analysis}

The STATA program version 8.2 (College Station, TX, USA) was used to perform all statistical analyses. The total aneuploidy rate was expressed as the sum of disomic and nullisomic spermatozoa for any autosomes (chromosomes 13,18 and 21) and sex chromosomes (X and Y chromosomes) divided by the total number of spermatozoa analysed. The DNA fragmentation rate was calculated as the number of spermatozoa with fragmented DNA divided by the total number of spermatozoa analysed. Total aneuploidy and DNA fragmentation rates among the three groups were compared by $3 \times 2$ chi-squared tests. A comparison was considered to be statistically significant if $P<0.05$. If there was a significant difference, partition of the chi-squared was performed to check which pair contributed significantly to the difference.

\section{RESULTS}

The age (mean \pm s.d.) of the 50 males in this study was $39.1 \pm 10.3$ years. Semen parameters (mean \pm s.d.) were: volume, $2.3 \pm 0.9 \mathrm{ml}$; concentration, $3.0 \pm 1.6$ million $\mathrm{ml}^{-1}$; motility, $32.6 \% \pm 6.5 \%$; and normal morphology, $8.0 \% \pm 3.3 \%$. The mean total motile sperm concentration ( \pm s.d.) after preparation was $2.2 \pm 1.1$ million $\mathrm{ml}^{-1}$, with a normal morphology (mean \pm s.d.) of $9.4 \% \pm 4.7 \%$.

The total number of spermatozoa analysed and the number of those with fragmentation and aneuploidy (sum of disomic and nullisomic spermatozoa for both autosomes and sex chromosomes) are shown in Table 1. In the control group, the aneuploidy rates for chromosomes
$13,18,21$ and sex chromosomes were $1.50 \%, 1.55 \%, 1.54 \%$ and $2.28 \%$, respectively, and the diploidy rate was $0.14 \%$.

When only the second-best spermatozoa were available for selection under high magnification and MSOME criteria, the sperm aneuploidy rate was significantly higher than that in the subgroup in which the best spermatozoa were selected. However, there was no significant difference in the DNA fragmentation rate between the two subgroups (Table 2).

\section{DISCUSSION}

Given a disomic frequency per chromosome of approximately $0.1 \%$ in normal spermatozoa, it has been estimated that at least 10000 spermatozoa must be scored to allow the detection of a doubling in the frequency of disomy, with a type I error of 0.05 and a power of $80 \% .^{21}$ However, this was not possible in the sperm group selected on high-magnification morphology, as the process was time- and labour-intensive. To overcome this problem, we recruited only males with severe oligozoospermia, as their spermatozoa are known to harbour a higher incidence of aneuploidy than those of the general population. ${ }^{22-24}$ Consequently, the prevalence of aneuploidy in the selected spermatozoa in our control group was as high as $7 \%$.

Previous publications ${ }^{25,26}$ have shown that density gradient centrifugation increases the isolation of spermatozoa with normal morphology and DNA integrity. However, our study demonstrated that the incidence of sperm aneuploidy and DNA fragmentation was still high in males with a sperm concentration of $<5 \times 10^{6}$ million $\mathrm{ml}^{-1}$ after the process of density gradient preparation. This suggested that a strategy for sperm selection, such as HA binding or high-magnification morphology, should be employed in this population to select competent spermatozoa before ICSI.

It is still unclear if HA binding selects spermatozoa with normal morphology. In a study involving normozoospermic semen samples, ${ }^{27}$ the authors demonstrated that HA binding had limited efficacy in selecting motile spermatozoa with normal morphology at high magnification, while another study in oligozoospermic samples found a much higher percentage of nuclear normality in HA-bound than HA-unbound spermatozoa. ${ }^{15}$ The discrepancy in the two studies

Table 2 Aneuploidy and DNA fragmentation rates in those who had the best or second-best spermatozoa for selection under high magnification+MSOME criteria

\begin{tabular}{lcc}
\hline & Best spermatozoa available & The second-best spermatozoa available \\
\hline Total number of spermatozoa analysed & 1217 & 528 \\
Spermatozoa with DNA fragmentation & 20 & 11 \\
DNA fragmentation rate (\%) & 1.6 & 2.0 \\
Spermatozoa with aneuploidy & 2 & 10 \\
Sperm aneuploidy rate (\%) & 0.2 & - \\
\hline
\end{tabular}

Abbreviation: MSOME, motile sperm organellar morphology examination.

*Chi-squared test with Yates' correction. 
could be due to many factors. One possibility is that most unbound spermatozoa in normal samples have normal morphology and they are still in the process of binding, but yet to be bound. Moreover, some morphologically abnormal spermatozoa could contain normal DNA and vice versa. ${ }^{24}$ Therefore, in this study we focused only on HAbound spermatozoa, and employed DNA fragmentation and chromosome aneuploidy as the final outcomes.

We confirmed previous studies that both HA binding and high-magnification morphology were very effective in selecting spermatozoa with lower DNA fragmentation and aneuploidy rate $^{12,15,22-24}$ We also provided a head-to-head comparison between the two methods of sperm selection, and showed that selection under high-magnification was better than HA-binding for low sperm DNA fragmentation. Despite the statistical difference, we are doubtful whether the DNA fragmentation rate of $2.6 \%$ vs $1.7 \%$ is clinically meaningful Moreover, there was no difference in the aneuploidy rates in the selected spermatozoa obtained from the two methods $(0.8 \%$ vs $0.7 \% ; P=0.583)$.

The limitation of this study was that fewer than 2000 spermatozoa were observed in the MSOME group, and the number of spermatozoa evaluated per individual was not uniform, depending on the availability of normal spermatozoa (at least 20 spermatozoa per sample). Thus the results might not accurately reflect the group as a whole, and could be influenced by the characteristics of those individuals in whom the higher number of spermatozoa were analysed. Moreover, no oocytes could be inseminated with the selected spermatozoa. Hence, there were no data on the fertilization, clinical pregnancy and miscarriage rates of embryos that originated from the selected spermatozoa in either group.

When only the second-best spermatozoa were available for selection under high-magnification, they showed no difference in the DNA fragmentation but a 10-fold difference in the incidence of sperm aneuploidy from the cases where the best spermatozoa were available. Although the number of analysed spermatozoa was small (1745), the data supported the results of a clinical study by Berkovitz et al. ${ }^{19}$ that the use of the best spermatozoa gave a fourfold increase in implantation rate and a threefold increase in clinical pregnancy rate over the use of the second-best spermatozoa.

In conclusion, both high-magnification morphology and HA binding served as excellent tools in the selection of spermatozoa with a $>90 \%$ reduction in DNA fragmentation and a $>88 \%$ reduction in aneuploidy rates; better than those from conventional selection by an embryologist. Some have even advocated the use of HA in combination with high-magnification morphology to increase the efficiency of sperm selection but our data did not support this. ${ }^{15}$ When time and cost are considered, we believe there is currently not enough evidence to indicate an advantage of high-magnification sperm selection or the combination of this method with HA-binding over that of HA-binding alone. More research on the cost-effectiveness of these two methods of sperm selection is urgently needed.

\section{AUTHOR CONTRIBUTIONS}

TV made substantial contributions to conception, design, analysis and interpretation of the data, and drafted the final manuscript. SM participated in its design, acquisition of data, data analysis and helped to draft the manuscript. All authors read and approved the final manuscript.

\section{COMPETING FINANCIAL INTERESTS}

All authors have no potential conflicts of interest, whether of a financial or other nature, with any pharmaceutical company.

\section{ACKNOWLEDGMENTS}

This research was supported by Phyathai Sriracha Hospital, Cholburi, Thailand (P1/2553). We would like to thank embryologists and colleagues at Phyathai Sriracha Hospital for their kind help and assistance during this study.

1 Tournaye H. Male factor infertility and ART. Asian J Androl 2012; 14: 103-8.

2 Twigg JP, Irvine DS, Aitken RJ. Oxidative damage to DNA in human spermatozoa does not preclude pronucleus formation at intracytoplasmic sperm injection. Hum Reprod 1998; 13: 1864-71.

3 Martin RH, Spriggs E, Rademaker AW. Multicolor fluorescence in situ hybridization analysis of aneuploidy and diploidy frequencies in 225,846 sperm from 10 normal men. Biol Reprod 1996; 54: 394-8.

4 Shi Q, Martin RH. Aneuploidy in human spermatozoa: FISH analysis in men with constitutional chromosomal abnormalities, and in infertile men. Reproduction 2001; 121: 655-66.

5 Gianaroli L, Magli MC, Cavallini G, Crippa A, Nadalini M et al. Frequency of aneuploidy in sperm from patients with extremely severe male factor infertility. Hum Reprod 2005; 20: 2140-52.

6 Tempest HG, Homa ST, Dalakiouridou M, Christopikou D, Wright D et al. The association between male infertility and sperm disomy: evidence for variation in disomy levels among individuals and a correlation between particular semen parameters and disomy of specific chromosome pairs. Reprod Biol Endocrinol. 2004; 2: 82.

7 Sun F, Ko E, Martin RH. Is there a relationship between sperm chromosome abnormalities and sperm morphology? Reprod Biol Endocrinol 2006; 4: 1.

8 Aitken RJ, de Luliis GN. Origins and consequences of DNA damage in male germ cells. Reprod Biomed Online 2007; 14: 727-33.

9 Bartoov B, Berkovitz A, Eltes F, Kogosovsky A, Yagoda A et al. Pregnancy rates are higher with intracytoplasmic morphologically selected sperm injection than with conventional intracytoplasmic injection. Fertil Steril 2003; 80: 1413-9.

10 Hazout A, Dumont-Hassan M, Junca AM, Cohen BP, Tesarik J. High-magnification ICSI overcomes paternal effect resistant to conventional ICSI. Reprod Biomed Online 2006; 12: 19-25.

11 Antinori M, Licata E, Dani G, Cerusico F, Versaci C et al. Intracytoplasmic morphologically selected sperm injection: a prospective randomized trial. Reprod Biomed Online 2008; 16: 835-41.

12 Huszar G, Ozenci CC, Cayli S, Zavaczki Z, Hansch E et al. Hyaluronic acid binding by human sperm indicates cellular maturity, viability, and unreacted acrosomal status. Fertil Steril 2003; 79 (Suppl 3): 1616-24.

13 Jakab A, Sakkas D, Delpiano E, Cayli S, Kovanci E et al. Intracytoplasmic sperm injection: a novel selection method for sperm with normal frequency of chromosomal aneuploidies. Fertil Steril 2005; 84: 1665-73.

14 Yagci A, Murk W, Stronk J, Huszar G. Spermatozoa bound to solid state hyaluronic acid show chromatin structure with high DNA chain integrity: an acridine orange fluorescence study. J Androl 2010; 31: 566-72.

15 Parmegiani L, Cognigni GE, Bernardi S, Troilo E, Ciampaglia W et al. 'Physiologic ICSI': hyaluronic acid (HA) favors selection of spermatozoa without DNA fragmentation and with normal nucleus, resulting in improvement of embryo quality. Fertil Steril 2010; 93: 598-604.

16 World Health Organization. WHO Laboratory Manual for the Examination and Processing of Human Semen. 5th ed. Geneva: WHO press; 2010.

17 Peer S, Eltes F, Berkovitz A, Yehuda R, Itsykson P etal. Is fine morphology of the human sperm nuclei affected by in vitro incubation at 37 degrees C? Fertil Steril 2007; 88: 1589-94.

18 Bartoov B, Berkovitz A, Eltes F, Kogosowski A, Menezo Y et al. Real-time fine morphology of motile human sperm cells is associated with IVF-ICSI outcome. $J$ Androl 2002; 23: 1-8.

19 Berkovitz A, Eltes F, Yaari S, Katz N, Barr I et al. The morphological normalcy of the sperm nucleus and pregnancy rate of intracytoplasmic injection with morphologically selected sperm. Hum Reprod 2005; 20: 185-90.

20 Vidal F, Moragas M, Catala V, Torello MJ, Santalo J et al. Sephadex filtration and human serum albumin gradients do not select spermatozoa by sex chromosome: a fluorescent in-situ hybridization study. Hum Reprod 1993; 8: 1740-3.

21 Martin R. Fluorescence in situ hybridization aficionados and rater variation. Fertil Steril 1995; 4: 217-8; author reply 219-20.

22 Garolla A, Fortini D, Menegazzo M, de Toni L, Nicoletti V et al. High-power microscopy for selecting spermatozoa for ICSI by physiological status. Reprod Biomed Online 2008; 17: 610-6.

23 Oliveira JB, Massaro FC, Baruffi RL, Mauri AL, Petersen CG et al. Correlation between semen analysis by motile sperm organelle morphology examination and sperm DNA damage. Fertil Steril 2010; 94: 1937-40.

24 Franco JG Jr, Baruffi RL, Mauri AL, Petersen CG, Oliveira JB et al. Significance of large nuclear vacuoles in human spermatozoa: implications for ICSI. Reprod Biomed Online 2008; 17: 42-5.

25 Sakkas D, Manicardi GC, Tomlinson M, Mandrioli M, Bizzaro D et al. The use of two density gradient centrifugation techniques and the swim-up method to separate spermatozoa with chromatin and nuclear DNA anomalies. Hum Reprod 2000; 15: 1112-6.

26 Tomlinson MJ, MoffattO, Manicardi GC, Bizzaro D, Afnan M etal. Interrelationships between seminal parameters and sperm nuclear DNA damage before and after density gradient centrifugation: implications for assisted conception. Hum Reprod 2001; 16: 2160-5.

27 Petersen CG, Massaro FC, Mauri A, Oliveira JBA, Baruffi RLR et al. Efficacy of hyaluronic acid binding assay in selecting motile spermatozoa with normal morphology at high magnification. Reprod Biol Endocrinol 2010; 8: 149. 